\title{
ACTIONS OF LINEARLY REDUCTIVE GROUPS ON PI-ALGEBRAS
}

\author{
NIKOLAUS VONESSEN
}

\begin{abstract}
Let $G$ be a linearly reductive group acting rationally on a PIalgebra $R$. We study the relationship between $R$ and the fixed ring $R^{G}$, generalizing earlier results obtained under the additional hypothesis that $R$ is affine.
\end{abstract}

Let $k$ be an algebraically closed field, $R$ a PI-algebra over $k$, and $G$ a linearly reductive affine algebraic group over $k$ acting rationally on $R$ by algebra automorphisms. In $\left[\mathrm{V}_{1}\right]$, we studied the relationship between $R$ and the fixed ring $R^{G}$ under the assumption that $R$ is affine, i.e., finitely generated as a $k$-algebra. In this paper we show that many of the results obtained there are actually true without the assumption that $R$ is affine. Since this paper is a sequel to $\left[\mathrm{V}_{1}\right]$, we will refer to the latter as [I]; the reference I.5.4 means then $\left[\mathrm{V}_{1}, 5.4\right]$.

The contents of this article are roughly as follows. Section 1 contains a few preliminaries. Sections 2 and 3 deal, respectively, with localization and prime ideals, assuming that the PI-algebra $R$ is either Noetherian or integral over its center. In $\S \S 4$ and 5 we look at actions by spec-inner automorphisms (i.e., automorphisms under which every prime ideal is stable). Here $R$ is an arbitrary PI-algebra. Finally, we prove in an appendix some "triviality" criteria for rational actions of connected groups.

Among the results we obtain are the following. Assume first that $R$ is onesided Noetherian. Many proofs in [I] used Gelfand-Kirillov dimension and Borho's theory of restricted extensions of algebras of finite GK-dimension. Replacing these tools by Krull dimension in the sense of Gabriel and Rentschler and by the theory of FBN rings, we can generalize various results of [I]. In particular, we obtain additivity principles for Goldie ranks and PI-degrees for the ring extension $R^{G} \subseteq R(2.2)$. These follow from a localizability result: If $P$ is a prime ideal of $R$, then $R^{G} / P \cap R^{G}$ has an Artinian total ring of fractions which embeds into the ring of fractions of $R / P$. In fact, if char $k=0$, then the Small set of $R^{G} / P \cap R^{G}$ (i.e., the set of those elements which are regular modulo the nilradical) consists of regular elements of $R / P$ and satisfies the Ore conditions for $R / P$. Most of these results remain true if $R$ is not Noetherian

\footnotetext{
Received by the editors February 7, 1990 and, in revised form, October 22, 1990.

1980 Mathematics Subject Classification (1985 Revision). Primary 16A74; Secondary 16A38, 16 A33.

Key words and phrases. PI-algebras, group actions, linearly reductive groups, Noetherian rings, inner automorphisms.

Partially supported by NSF grant DMS 8901491 .
} 
but instead integral over its center $(2.5,2.6)$. In this case one can even get away with a localization by fixed points in the center of $R$. However, the resulting localization of $R^{G}$ is only Artinian modulo a nilpotent ideal.

Next, we look at the behavior of prime ideals in the ring extension $R^{G} \subseteq R$. Given a prime ideal $P$ of $R$, we denote by $\Phi(P)$ the set of all prime ideals of $R^{G}$ minimal over $P \cap R^{G}$. Since $R$ is a PI-algebra, $\Phi(P)$ is always a finite set (cf. I.6.3). So $\Phi: \operatorname{Spec} R \multimap \operatorname{Spec} R^{G}$ is a finite-valued correspondence. If $R$ is one-sided Noetherian or integral over its center, $\Phi$ is surjective, i.e., lying over holds: For every prime ideal $p$ of $R^{G}$, there is a prime ideal $P$ of $R$ such that $p$ is minimal over $P \cap R^{G}$ (3.2). Further results in $\S 3$ deal with the question of how far the correspondence $\Phi$ is from being a map $(3.4,3.5,3.6)$. Particularly nice results are obtained in case $R$ is a (not necessarily Noetherian) Azumaya algebra.

Most of the results mentioned so far depend heavily on the fact that $R$ is Noetherian or integral over its center, even if $R$ is affine, as examples in $\S I .5$ show. It is in general also not possible to relax the hypothesis that $G$ be linearly reductive (see $\S$ I.8).

We then turn to actions by spec-inner automorphisms, i.e., automorphisms under which all prime ideals are stable (see $\S$ I.9.1). The ring $R$ is now an arbitrary PI-algebra over $k$. Let $H$ be a connected linear algebraic group acting rationally on $R$. If $R$ is semiprime and $H$ acts by spec-inner automorphisms, then every element in the center of $R$ is fixed (4.1). And under certain conditions, if $R$ is prime, then $H$ acts by spec-inner automorphisms iff it acts by X-inner automorphisms (i.e., by automorphisms which fix the center of $R$ pointwise): Namely, this is true if either $H$ is reductive, or if $R$ is Noetherian, or if $R$ is integral over its center (4.2). One way of looking at this result is that in these cases, $\mathrm{X}$-inner actions are "functorial." Namely, if $H$ acts under these hypotheses by X-inner automorphisms, then $H$ acts also on $R / P$ by X-inner automorphisms for every prime ideal $P$ of $R$.

Since we are concerned with actions of linearly reductive groups, Proposition 4.2 shows that for our purposes actions by spec-inner and X-inner automorphisms are more or less the same in the prime case. For an arbitrary PI-algebra $R$, however, these notions differ: If $H$ is reductive, it acts by spec-inner automorphisms iff it acts by $\mathrm{X}$-inner automorphisms on $R / P$ for every minimal prime $P$ of $R$ (4.2). If, however, $R$ is semiprime with a finite number of minimal prime ideals, then $H$ acts by $\mathrm{X}$-inner automorphisms iff there is some minimal prime ideal $P$ of $R$ such that it acts by $\mathrm{X}$-inner automorphisms on $R / P$ (I.9.1.6).

After these preliminaries, we study in $\S 5$ chain conditions and the behavior of prime ideals in the extension $R^{G} \subseteq R$ for a rational action of a linearly reductive group $G$ by spec-inner automorphisms. In particular, we show that $R^{G}$ is semiprime if $R$ is (5.1), and prove a lying over result (5.4).

In the appendix, we prove some "triviality" criteria for rational actions of a connected linear algebraic group $H$ on a $k$-algebra $A$. Namely, if $A$ has too many invertible elements, the induced action on the center of $A$ has to be trivial. To be more precise, this is true if $A$ is a prime PI-algebra with nonzero Jacobson radical, or a division algebra. In the latter case, the action is actually trivial on all of $A$, provided that either $A$ is commutative, or char $k=0$, 
or $H$ is reductive. These results should be well known, and might even be "intuitively obvious," but proofs are included for lack of a reference. I am grateful to R. Guralnick for helping me with parts of the proofs.

Finally, I would like to thank A. Braun for many interesting and helpful conversations.

\section{Preliminaries}

In this section, we make a few introductory observations. A look into $§ I .2$ might also be helpful; the facts collected in I.2.1-13 will be used frequently.

If $R$ is left Noetherian, then so is the fixed ring $R^{G}$. More generally, if $R$ is a finite left module over a left Noetherian, $G$-stable subalgebra $S$, then $R^{G}$ is a Noetherian $S^{G}$-module (see I.4.1). And if $R$ is integral over a central, $G$-stable subalgebra $C$, then $R^{G}$ is integral over $C^{G}$ : Given $r \in R^{G}$, there are some $c_{i} \in C$ such that $r^{n}+c_{n-1} r^{n-1}+\cdots+c_{0}=0$. Applying the Reynolds operator $\downarrow: R \rightarrow R^{G}$ (see I.2.10), we get $r^{n}+\emptyset\left(c_{n-1}\right) r^{n-1}+\cdots+\emptyset\left(c_{0}\right)=0$. Since $\natural(C)=C^{G}$, the assertion about integrality follows.

We will use repeatedly that in case $R$ is prime, the action of $G$ on $R$ extends to a rational action on the trace ring $\mathrm{T}(R)$ of $R$ (I.3.4).

The following simple but useful observation is already implicitly contained in I.9.3.1.

Lemma 1.1. If $C$ is a central $G$-stable subalgebra of $R$, and $p \in \Phi(P)$, then $P \cap C^{G}=p \cap C^{G}$.

Proof. We will prove the following more general statement: Let $A \subset B$ be rings, let $P$ be a prime of $B$, and let $p$ be a prime of $A$ minimal over $P \cap A$. Then $(P \cap A) \cap C=p \cap C$ for every central subalgebra $C$ of $B$.

Assume that $(P \cap A) \cap C \subsetneq p \cap C$. Factoring out by $P$, we may therefore assume that $B$ is prime and that $p$ is a minimal prime ideal of $A$ which contains a nonzero central element $x$ of $B$. Note that $x$ is both central and regular in $A$. This is impossible by [R, 5.2.11].

Remark 1.2. Chin [C] proved recently that if a connected linear algebraic group $H$ acts rationally on an associative $k$-algebra, then $H$-prime ideals are prime, or equivalently, all minimal prime ideals are $H$-stable. In our setup this implies the following. Let $P$ be a prime ideal of $R$. Set $I=\bigcap_{g \in G} P^{g}$ and $I_{0}=\bigcap_{g \in G^{\circ}} P^{g}$, where $G^{\circ}$ denotes the connected component of $G$. Then $I_{0}$ is prime, and $I$ is the intersection of the finitely many $G$-conjugates of $I_{0}$. In particular, there are only a finite number of prime ideals in $R$ which are minimal over $I$, and for any such minimal prime $Q, Q \cap R^{G}=P \cap R^{G}=I \cap R^{G}$. We will use these facts quite heavily when we study actions on algebras which are integral over their centers (see $\S 3$ and Proposition 4.2).

Finally, let us mention one of the problems one encounters if one works with PI-algebras which are not necessarily affine: If $M$ is a maximal ideal of $R$, it is not clear that $\Phi(M)$ consists of maximal ideals of $R^{G}$. This is true, however, if $R$ is affine or if $G$ acts by spec-inner automorphisms (see $\S 4$ ): If $M$ is a maximal ideal of $R$, then $R^{G} / M \cap R^{G}$ embeds into the simple algebra $R / M$. In the first case, $R / M$ is finite dimensional over $k$, and in the second, $R^{G} / M \cap R^{G}$ is the fixed ring of the Artinian algebra $R / M$. In either case, $R^{G} / M \cap R^{G}$ is Artinian and $\Phi(M)$ consists only of maximal ideals. 


\section{AdDITIVITY PRINCIPLES AND LOCALIZATION}

In most results of this section we will assume that $R$ is left Noetherian (the exceptions are Proposition 2.5 and Lemma 2.6). Recall that if $R$ is left Noetherian, then so is $R^{G}$. And if $R$ is left Noetherian and semiprime, then it is in fact two-sided Noetherian by Cauchon's theorem.

An important tool in $\S \mathbf{I} .5$ was Borho's localization theory for Noetherian algebras of finite Gelfand-Kirillov dimension [B]. However, nonaffine PI-algebras can have infinite Gelfand-Kirillov dimension. But many arguments in $\S I .5$ can be adapted, if one uses instead Krull dimension in the sense of Gabriel and Rentschler, and the theory of FBN rings (cf. [MR, Chapter 6]). We remark that Noetherian PI-algebras are FBN rings, and that FBN rings satisfy the "bimodule condition" [MR, 4.5.7 and 6.4.13]. This allows application of several localization results due to Warfield [W] (see [MR, §4.5]).

Lemma 2.1. Assume that the characteristic of $k$ is zero, and that $R$ is (left) Noetherian and semiprime. Then $R$ is bi-restricted as an $R^{G}$-module, i.e., the principal bimodule $R^{G} x R^{G}$ is Noetherian both as a left and right $R^{G}$-module for all $x \in R$.

I do not know if this result is true in prime characteristic.

Proof. Using the fact that in characteristic zero the trace ring of a prime Noetherian PI-algebra is finite over its center, the proof of I.5.4 goes through.

Denote Krull dimension in the sense of Gabriel and Rentschler and Goldie rank by Kdim and rk, respectively. Later on we will also use ordinal-valued, classical Krull dimension, denoted by dim (see [MR, 6.4.3]).

Proposition 2.2. Assume that $R$ is left Noetherian. Let $P$ be a prime ideal of $R$, and denote by $\Phi(P)=\left\{p_{1}, \ldots, p_{n}\right\}$ the primes of $R^{G}$ minimal over $P \cap R^{G}$.

(a) The algebra $R^{G} / P \cap R^{G}$ has an Artinian total ring of quotients which embeds into the total ring of quotients of $R / P$. Moreover, the algebra $A$ generated by $R / P$ and $\mathrm{Q}\left(R^{G} / P \cap R^{G}\right)$ is prime and Noetherian. And if chark $=0$, then the Small set $S$ of $R^{G}$ satisfies the Ore conditions for $R / P$, so that $A=(R / P) S^{-1}$ is a localization of $R / P$.

(b) (Equidimensionality) For all $i, \operatorname{Kdim} R^{G} / p_{i}=\operatorname{Kdim} R^{G} / P \cap R^{G}$.

(c) (Additivity principles) There are positive integers $z_{i}$ such that $\operatorname{rk}(R / P)=$ $\sum z_{i} \operatorname{rk}\left(R^{G} / p_{i}\right)$. A similar statement holds for PI-degrees.

None of the statements in parts (a) and (b) is true if $R$ is not one-sided Noetherian (see I.5.6 and I.5.13). I do not know if the set $S$ in (a) satisfies the Ore conditions for $R / P$ also in prime characteristic. In characteristic zero, the proposition is essentially a corollary to Lemma 2.1 . We prove it first in this case. Maybe one should note here that the only connected linearly reductive groups in prime characteristic are the tori (cf. the discussion in $§ I .0$ ).

Proof of Proposition 2.2 in characteristic zero. (a) follows more or less immediately from [MR, 4.5.9]. That $S$ satisfies the Ore conditions for $R / P$ follows from regularity by the localizability criterion $[\mathrm{B}, 5.1]$. This implies that $A=(R / P) S^{-1}$ is Noetherian.

(b) Using [MR, 4.5.4 and 4.5.6], one sees that for all $i$ and $j$ there is an $\left(R^{G} / p_{i}\right)-\left(R^{G} / p_{j}\right)$-bimodule which is finitely generated and torsion free on each 
side. Hence [MR, 6.4.13] implies that $\mathrm{Kdim} R^{G} / p_{i}=\mathrm{Kdim} R^{G} / p_{j}$. It follows now by [MR, 6.3.8] that $\mathrm{Kdim} R^{G} / p_{i}=\mathrm{Kdim} R^{G} / P \cap R^{G}$.

(c) The part about Goldie rank is contained in the already quoted theorem [MR, 4.5.9], and [BS, 7.3] gives an additivity principle for PI-degrees for $\mathrm{Q}\left(R^{G} / P \cap R^{G}\right) \subseteq \mathrm{Q}(R / P)$, implying the result.

Before we give a characteristic-free proof of Proposition 2.2, we need a technical result.

Lemma 2.3. Let $A \subseteq B$ be a (left and right) finite extension of $F B N$ rings such that for all minimal prime ideals $q$ of $B, \operatorname{Kdim} B / q=\mathrm{Kdim} B$.

(a) For all minimal prime ideals $p$ of $A, \mathrm{Kdim} A / p=\mathrm{K} \operatorname{dim} A$.

(b) If $q$ is a minimal prime ideal of $B$, and $p$ is a prime ideal of $A$ minimal over $q \cap A$, then $p$ is a minimal prime ideal of $A$.

(c) If $B$ has an Artinian total ring of quotients $\mathrm{Q}(B)$, then also $A$ has an Artinian total ring of quotients $\mathrm{Q}(A)$, and $\mathrm{Q}(A)$ embeds into $\mathrm{Q}(B)$. Moreover, then $\mathrm{Q}(B)=B \mathrm{Q}(A)$.

Proof. (a) Note first that $p$ contains $q \cap A$ for some minimal prime $q$ of $B$. Hence $\mathrm{Kdim} A / p=\mathrm{Kdim} A / q \cap A$ since $A \subseteq B$ is a bi-restricted extension of FBN rings (cf. the characteristic zero proof of 2.2(b) above). Therefore $\mathrm{K} \operatorname{dim} A / p=\mathrm{K} \operatorname{dim} A / q \cap A=\mathrm{K} \operatorname{dim} B / q=\mathrm{K} \operatorname{dim} B=\mathrm{K} \operatorname{dim} A$, where the second and fourth equality follow by [MR, 6.4.13].

(b) Suppose that $p$ is not a minimal prime of $A$. Then $p$ contains strictly a minimal prime $p_{0}$ of $A$, and $\operatorname{Kdim} A \geq \operatorname{Kdim} A / p_{0}>\operatorname{Kdim} A / p=\operatorname{Kdim} A$, a contradiction.

(c) Let $x$ be an element of $A$ which is regular modulo the nilradical $N$ of $A$. Let $q$ be any minimal prime of $B$, and let $p_{1}, \ldots, p_{n}$ be the primes of $A$ minimal over $q \cap A$. By (b), the $p_{i}$ are minimal prime ideals of $A$. Set $N^{\prime}=p_{1} \cap \cdots \cap p_{n}$. Then $x$ is regular modulo $N^{\prime}$. Now by [MR, 4.5.9], $A / q \cap A$ has an Artinian total ring of fractions which embeds into $B / q$. In particular, $x$ is regular in $B / q$. Since $q$ was an arbitrary minimal prime of $B$, it follows that $x$ is regular in $B$ modulo the nilradical of $B$. Since $\mathrm{Q}(B)$ exists, Small's theorem [MR, 4.1.4] implies that $x$ is regular in $B$. A second application of this theorem shows that $A$ has an Artinian total ring of quotients which embeds into $\mathrm{Q}(B)$. Now [B, 5.1] shows that the Small set of $A$ satisfies the Ore conditions for $B$. Hence $B \mathrm{Q}(A)$ is an algebra, and an Artinian $\mathrm{Q}(A)$ module. Therefore $\mathrm{Q}(B)=B \mathrm{Q}(A)$.

Proof of Proposition 2.2 in arbitrary characteristic. We note that (c) is a consequence of (a), using [JS] and [BS, 7.3]. We assume first that $G$ is connected. Factoring out by the prime ideal $\bigcap_{g \in G} P^{g}$, we may hence assume that $\bigcap_{g \in G} P^{g}=0$. In particular, $R$ is now prime, $P \cap R^{G}=0$, and the $p_{i}$ are the minimal prime ideals of $R^{G}$. We proceed in several steps.

Let $Q$ be a prime ideal of the trace ring $\mathrm{T}(R)$ lying over $P$. (Actually, $Q$ is unique.) Then also $\bigcap_{g \in G} Q^{g}=0$ and $Q \cap \mathrm{T}(R)^{G}=0$. Note that $\mathrm{Q}(R / P)$ embeds into $\mathrm{Q}(\mathrm{T}(R) / Q)$. Now $\mathrm{T}(R)^{G}$ embeds into $\mathrm{T}(R) / Q$. The nonzero elements of $\mathrm{Z}(\mathrm{T}(R))^{G}$ are central in $\mathrm{T}(R) / Q$ and thus regular. Setting $S=\mathrm{Z}(\mathrm{T}(R))^{G} \backslash\{0\}, \mathrm{T}(R)^{G} S^{-1}$ is an integral centralizing extension of 
the field $\mathrm{Q}\left(\mathrm{Z}(\mathrm{T}(R))^{G}\right)$ and thus has classical Krull dimension zero. Hence $\mathrm{T}(R)^{G} S^{-1}=\mathrm{Q}\left(\mathrm{T}(R)^{G}\right)$, and this algebra embeds into $\mathrm{Q}(\mathrm{T}(R) / Q)$.

Next we show that for a minimal prime $q$ of $\mathrm{T}(R)^{G}, \operatorname{Kdim} \mathrm{T}(R)^{G} / q=$ $\operatorname{Kdim} \mathrm{T}(R)^{G}$. Note that $q \cap \mathrm{Z}(\mathrm{T}(R))^{G}=0$. Since $\mathrm{Z}(\mathrm{T}(R))^{G} \subseteq \mathrm{T}(R)^{G} / q$ is an integral centralizing extension of PI-rings, these rings have the same classical Krull dimension (see [S, p. 247]). Since classical Krull dimension and Krull dimension in the sense of Gabriel and Rentschler coincide for FBN rings (see [MR, 6.4.8]), $\mathrm{Kdim} \mathrm{T}(R)^{G} / q$ is independent of the particular minimal prime $q$. Therefore it is equal to $\operatorname{Kdim} \mathrm{T}(R)^{G}$, (cf. [MR, 6.3.8]).

Lemma 2.3 applied to $R^{G} \subseteq \mathrm{T}(R)^{G}$ implies now that $R^{G}$ has an Artinian ring of fractions $\mathrm{Q}\left(R^{G}\right)$, and that $\mathrm{Q}\left(\mathrm{T}(R)^{G}\right)=\mathrm{T}(R)^{G} \mathrm{Q}\left(R^{G}\right)$. Since the regular elements of $R^{G}$ are regular in $\mathrm{T}(R)^{G}$ and thus also in $\mathrm{T}(R) / Q, \quad \mathrm{Q}\left(R^{G}\right)$ embeds into $\mathrm{Q}(R / P)$. The algebra generated by $\mathrm{Q}\left(R^{G}\right)$ and $\mathrm{T}(R) / Q$ is actually a localization of $\mathrm{T}(R) / Q$ and as such Noetherian. Since this algebra is a finite centralizing extension of the algebra $A$ generated by $\mathrm{Q}\left(R^{G}\right)$ and $R / P, A$ is Noetherian, completing the proof of (a). Part (b) also follows from Lemma 2.3.

So we have proved the proposition in the case when $G$ is connected. If $G$ is not connected, we consider the ring extensions $R^{G} / P \cap R^{G} \subseteq R^{G^{\circ}} / P \cap R^{G^{\circ}} \subseteq$ $R / P$ (recall I.2.9). Parts (a) and (b) follow now easily from the connected case by the lemma.

We now improve slightly on Proposition 2.2(a).

Corollary 2.4. Suppose that $R$ is (left) Noetherian and semiprime, and that $\operatorname{Min} R^{G}=\Phi(\operatorname{Min} R)$. The latter means that for all minimal prime ideals $P$ of $R$ and for all $p \in \Phi(P), p$ is a minimal prime ideal of $R^{G}$. Then the Small set $S$ of $R^{G}$ consists of regular elements in $R$. Hence the total ring of fractions $\mathrm{Q}\left(R^{G}\right)$ exists, is Artinian, and is contained in the total ring of fractions $\mathrm{Q}(R)$ of $R$. Moreover, the algebra generated by $\mathrm{Q}\left(R^{G}\right)$ and $R$ is Noetherian. Finally, if char $k=0$, then $S$ is a left and right Ore set for $R$.

This result will be used in the proof of Theorem 3.4. Concerning the condition " $\operatorname{Min} R^{G}=\Phi(\operatorname{Min} R)$ " see I.5.11. Again, this result is not true if $R$ is not Noetherian (see I.5.13).

Proof. Set $A=R^{G}$ and $B=R$, and let $P_{1}, \ldots, P_{n}$ be the minimal prime ideals of $B$. By Proposition 2.2(a), the Small set $S_{i}$ of $A / P_{i} \cap A$ is regular in $B / P_{i}$. The assumption on minimal ideals implies that the Small set $S$ of $A$ is regular in $A / P_{i} \cap A$ (cf. the proof of I.5.10). Hence $S$ is regular in $\bigoplus B / P_{i}$ and thus in $B$. Thus $\mathrm{Q}(A)$ exists, is Artinian, and embeds into $\mathrm{Q}(B)$. Denote by $C_{i}$ the subalgebra of $\mathrm{Q}\left(B / P_{i}\right)$ generated by $B / P_{i}$ and $\mathrm{Q}\left(A / P_{i} \cap A\right)$. By Proposition 2.2(a), each $C_{i}$ is Noetherian. Note that $\mathrm{Q}\left(A / P_{i} \cap A\right)$ is a homomorphic image of $\mathrm{Q}(A)$. Hence $\oplus C_{i}$ is the subalgebra of $\oplus \mathrm{Q}\left(B / P_{i}\right)$ generated by $\bigoplus B / P_{i}$ and $\mathrm{Q}(A)$. If $C$ denotes the subalgebra of $\mathrm{Q}(B)$ generated by $B$ and $\mathrm{Q}(A)$, then $C \subseteq \oplus C_{i}$ is a finite central extension. Hence $C$ is Noetherian by the theorem of Eisenbud and Eakin. Finally, if char $k=0, A \subseteq B$ is birestricted by Lemma 2.1, and Borho's localizability criterion [B, 5.1] shows that $S$ satisfies the Ore conditions for $B$.

Under suitable hypotheses, one can even localize at central fixed points. This will be important in the proofs of Theorems 3.4, 3.5, and 3.6. 
Proposition 2.5. Assume that $R$ is a semiprime, not necessarily Noetherian PIalgebra with a finite number of minimal prime ideals. Suppose additionally that $R$ is integral over its center $C$, and that for all minimal prime ideals $P$ of $R$, $P \cap C^{G}$ is a minimal prime ideal of $C^{G}$. Denote by $S$ the set of regular elements of $C^{G}$. Then $S$ consists of regular elements of $R$, and the central localization $R^{G} S^{-1}$ is Artinian modulo its nilpotent nilradical.

As examples show, $R^{G} S^{-1}$ itself need not be Artinian. In fact, $R^{G}$ need not even have an Artinian total ring of fractions, even if $R$ is a prime Azumaya algebra.

Proof. Let $x \in S$. Then $x \notin P \cap C^{G}$ for all minimal prime ideals $P$ of $R$. Since all minimal prime ideals of $C$ are of the form $P \cap C, x$ does not belong to any minimal prime ideal of $C$. Hence $x$ is regular in $R$ (see [R, 1.7.17]). Thus $S$ consists of regular elements of $R$. Since $R$ has only a finite number of minimal prime ideals, so does its subring $C^{G}$. Hence the localization $C^{G} S^{-1}$ is semisimple Artinian. Note that $R^{G} S^{-1}$ has a finite number of minimal prime ideals and a nilpotent nilradical (see, e.g., the proof of I.6.3). Since it is integral over $C^{G} S^{-1}$, its classical Krull dimension is zero. Hence it is semisimple Artinian modulo its nilradical.

For completeness, we record the following simple result.

Lemma 2.6. Suppose that $R$ is integral over its center. Then given a prime ideal $P$ of $R$, there are additivity principles for Goldie ranks and PI-degrees for $R^{G} / P \cap R^{G} \subseteq R / P$ (see 2.2).

Proof. Denote by $S$ the nonzero elements of $C^{G} / P \cap C^{G}$. They are central in $R / P$ and thus regular in $R / P$. One sees as in the above proof that $\left(R^{G} / P \cap R^{G}\right) S^{-1}$ is Artinian modulo a nilpotent ideal, and that it embeds into $\mathrm{Q}(R / P)$. The lemma follows now from the additivity principles of Joseph and Small [JS] and Bergman and Small [BS]. (The proof in [JS] works also in the case when the smaller ring is only Artinian modulo a nilpotent ideal; alternatively, see [MR, 4.5.4].)

\section{THE CORRESPONDENCE $\Phi$}

In this section we study the correspondence $\Phi: \operatorname{Spec} R \multimap \operatorname{Spec} R^{G}$ in the case when $R$ is either Noetherian or integral over its center. We first prove that $\Phi$ is surjective, i.e., that lying over holds for the ring extension $R^{G} \subseteq R$ (3.2). We then consider the question of how far the correspondence $\Phi$ is from being a map, obtaining a criterion involving PI-degrees (3.4). This allows interesting applications to Azumaya algebras $(3.5,3.6)$.

We start with an elementary lemma which is doubtlessly well known. I am grateful to L. W. Small for showing me the following very short proof.

Lemma 3.1. In a prime PI-ring, prime ideals of maximal PI-degree do not contain nonzero idempotents.

Proof. Let $P$ be a prime ideal of a prime PI-ring $R$ such that PIdeg $R / P=$ PIdeg $R$. Let $S$ be the complement of $P \cap \mathrm{Z}(R)$ in $\mathrm{Z}(R)$. Then $R S^{-1}$ is a local ring (cf. $[R, 1.9 .18])$ with Jacobson radical $P S^{-1}$. Since Jacobson radicals do not contain nonzero idempotents, neither does $P$. 
Theorem 3.2 (Lying over). Assume that $R$ is either left Noetherian or else integral over its center. Then given a prime ideal $p$ of $R^{G}$, there is a prime ideal $P$ of $R$ lying over $p$. That is, $p$ is minimal over $P \cap R^{G}$. Moreover, if $p$ is a maximal ideal of $R^{G}$, then $P$ can be chosen to be maximal.

The theorem says that the correspondence $\Phi$ is surjective and that $\operatorname{Max} R^{G} \subseteq$ $\Phi(\operatorname{Max} R)$. If $R$ is affine, the other inclusion also holds (cf. the discussion at the end of $\S 1)$. It seems unlikely that this is true for arbitrary Noetherian PIalgebras.

Proof. Let $p$ be a prime ideal of $R^{G}$. We want to show that there is a prime ideal $P$ of $R$ lying over $p$. We will proceed in several steps, proving first some special cases. In the case when $R$ is left Noetherian, we can certainly factor out by the nilpotent nilradical of $R$. Hence in this case we may assume that $R$ is semiprime and thus two-sided Noetherian by Cauchon's theorem.

Step 1. We assume here that $R$ is integral over a central, $G$-invariant subalgebra $C$. Then $R^{G}$ is integral over $C^{G}$. We can construct as in I.7.11, Step 1, a prime ideal $P$ of $R$ such that $P \cap R^{G} \subseteq p$ and $P \cap C^{G}=p \cap C^{G}$. Incomparability for the integral centralizing extension $C^{G} \subseteq R^{G}$ shows now that $p$ is minimal over $P \cap R^{G}$.

Step 2. We assume now that $G$ is connected and $R$ is Noetherian. Let $P$ be a $G$-stable ideal of $R$ maximal with respect to the property $P \cap R^{G} \subseteq p$. Then $P$ is $G$-prime and hence prime since $G$ is connected (cf. I.2.19). We will show that $p$ is minimal over $P \cap R^{G}$. Factoring out by $P$, we may assume that $R$ is prime, and that for every nonzero $G$-stable ideal $I$ of $R, I \cap R^{G} \nsubseteq p$.

Suppose that $p$ is not minimal. Then $p$ contains strictly a minimal prime $p_{0}$. Denote by $\mathrm{T}(R)$ the trace ring of $R$. Since $R^{G} \subseteq \mathrm{T}(R)^{G}$ is a finite extension of Noetherian PI-algebras, Letzter's going up and incomparability theorems [L, 4.8 and 2.4] yield prime ideals $q_{0} \subsetneq q$ of $\mathrm{T}(R)^{G}$ lying over $p_{0}$ and $p$, respectively. (A PI-theoretic proof of Letzter's lying over theorem is given in $\left[\mathrm{V}_{2}\right.$ ]; see also [BV] for a more general result for PI-rings.) By the first step, there is a prime $Q$ of $\mathrm{T}(R)$ lying over $q$. Since $q$ is minimal over $Q \cap \mathrm{T}(R)^{G}, q_{0}$ cannot contain $Q \cap \mathrm{T}(R)^{G}$. In particular, $Q \cap \mathrm{T}(R)^{G} \neq 0$. Let $I=\bigcap_{g \in G} Q^{g}$. Then $I$ is a $G$-stable prime ideal of $\mathrm{T}(R)$. Moreover, $I \cap \mathrm{T}(R)^{G}=Q \cap \mathrm{T}(R)^{G} \neq 0$, so that $I$ is nonzero. Hence $I \cap R$ is a nonzero $G$-stable prime ideal of $R$, but

$$
(I \cap R) \cap R^{G}=\left(I \cap \mathrm{T}(R)^{G}\right) \cap R^{G} \subseteq q \cap R^{G} \subseteq p,
$$

a contradiction.

Step 3. Now we prove lying over for $R$ Noetherian. Denote by $G^{\circ}$ the connected component of $R$. Recall I.2.8(a) and I.2.9. Factoring out $R$ by a $G$-stable ideal $I$ maximal with respect to the property that $I \cap R^{G} \subseteq p$, we may assume that $R$ is $G$-prime and that for every nonzero $G$-stable ideal $I$ of $R, I \cap R^{G} \nsubseteq p$. Let $P$ be a minimal prime of $R$. Then $\bigcap_{g \in G} P^{g}=0$, so that $P \cap R^{G}=0$. Therefore $P \cap R^{G} \subseteq p$. We will show that $p$ is a minimal prime ideal of $R^{G}$. If not, let $p_{0}$ be a minimal prime ideal of $R^{G}$ strictly contained in $p$. Using Montgomery's lying over result $\left[\mathrm{M}_{2}\right]$ (see also $\left[\mathrm{M}_{3}\right]$ ) for actions of finite groups, we can find a prime ideal $q_{0}$ of $R^{G^{\circ}}$ lying over $p_{0}$. Since $\bigcap_{\bar{x} \in G / G^{\circ}}\left(P \cap R^{G^{\circ}}\right)^{\bar{x}}=0$, there 
is some $x \in G$ such that $q_{0} \supseteq\left(P \cap R^{G^{\circ}}\right)^{x}=P^{x} \cap R^{G^{\circ}}$. Replacing $P$ b: $P^{x}$, we may assume that $P \cap R^{G^{\circ}} \subseteq q_{0}$. Using lying over for $R^{G} \subseteq R^{G^{\circ}}$ again, we find a prime ideal $q$ of $R^{G^{\circ}}$ lying over $p$ and containing $q_{0}$. Now let $Q$ be a prime ideal of $R$ lying over $q$ and containing $P$. Set $I=\bigcap_{g \in G} Q^{g}$. Then $I \cap R^{G}=\left(Q \cap R^{G^{\circ}}\right) \cap R^{G} \subseteq q \cap R^{G} \subseteq p$. Hence $I=0$. It follows easily that $P=\bigcap_{g \in G^{\circ}} Q^{g}$, so that $Q \cap R^{G^{\circ}}=P \cap R^{G^{\circ}} \subseteq q_{0} \subsetneq q$, a contradiction to $q$ being minimal over $Q \cap R^{G^{\circ}}$. This completes the proof of lying over for prime ideals in the case $R$ is Noetherian.

Step 4. Now we prove lying over for maximal ideals, assuming that $G$ is connected. Let $p$ be a maximal ideal of $R^{G}$. As already seen, there is a prime ideal $P$ of $R$ lying over $p$. We may assume that $P$ is $G$-stable. (In case $R$ is integral over its center, we use here again Chin's result, see 1.2.) We show first that $R^{G} / P \cap R^{G}$ has classical Krull dimension zero. If $R$ is Noetherian, this follows by equidimensionality. If $R$ is integral over its center $C$, we argue as follows: By lying over and incomparability for the integral centralizing extension $C^{G} \subseteq R^{G}$, it follows that $p \cap C^{G}$ is a maximal ideal of $C^{G}$. Hence $R / P \cap R^{G}$ is integral over the field $C^{G} / P \cap C^{G}=C^{G} / p \cap C^{G}$ and thus has dimension zero.

Using Lemma 3.1, one sees that the proof of I.7.4 actually works for arbitrary PI-rings with zero Jacobson radical. Hence if the Jacobson radical of $R / P$ is zero, I.7.4 yields a maximal ideal $M$ such that $p$ is minimal over $M \cap R^{G}$. And if the Jacobson radical of $R / P$ is nonzero, then the center of $R / P$ is pointwise fixed under the action of $G$ (Corollary A.2 in the appendix). The result now follows easily from 4.2(a) and 5.4 below: By the first result, $G$ acts on $R / P$ by spec-inner automorphisms. If now $M$ is a maximal ideal of $R$ containing $P$, then $\Phi(M) \cap \Phi(P) \neq \varnothing$ (since $\left.\operatorname{dim} R^{G} / P \cap R^{G}=0\right)$. Hence by 5.4, $M$ is equal to $P$ and thus lies over $p$.

Step 5. Finally, we prove lying over for maximal ideals. If $p$ is a maximal ideal of $R^{G}$, we first choose-by what we already have done-a $G$-prime ideal $I$ of $R$ lying over $p$. Factoring out by $I$, we may assume that $p$ is also a minimal prime ideal of $R^{G}$. Using Montgomery's result and Step 4, we then find a maximal ideal $M$ of $R$ such that $M \cap R^{G} \subseteq p$. Since $p$ is minimal, $M$ lies over $p$. This completes the proof of Theorem 3.2.

We now turn to the question of how far $\Phi$ is from being a map. The first step is given by a generalization of Lemma I.6.8 to PI-algebras which are not necessarily affine or Noetherian.

Lemma 3.3. Let $P_{1}$ and $P_{2}$ be prime ideals of $R$ with the same PI-degree $d$ such that $\Phi\left(P_{1}\right) \cap \Phi\left(P_{2}\right) \neq \varnothing$. Then $\overline{P_{1} \cdot G} \cap \overline{P_{2} \cdot G} \neq \varnothing$ by 1.6.12. Suppose that $\overline{P_{1} \cdot G} \cap \overline{P_{2} \cdot G}$ contains a prime ideal of PI-degree $d$. If additionally $R^{G}$ is Artinian modulo a nilpotent ideal, then $\Phi\left(P_{1}\right)=\Phi\left(P_{2}\right)$.

Here $P_{1} \cdot G$ denotes the $G$-orbit of $P_{1}$ in the spectrum of $R$, and $\overline{P_{1} \cdot G}$ denotes its closure in the Zariski topology.

Proof. We remark first that Lemma I.6.6 is true for rings which are only Artinian modulo a nilpotent ideal, since idempotents modulo nilpotent ideals can be lifted. (Note the misprints in I.6.6 and I.6.8: It should be $\sqrt{I} \nsubseteq \sqrt{J}$ and 
$\sqrt{P_{1} \cap R^{G}} \nsubseteq \sqrt{P_{2} \cap R^{G}}$, respectively.) The proof of Lemma I.6.8 now goes through with minor changes, using 3.1 instead of I.6.7. The only tricky point is why the prime ideal $M$ contains a prime $Q$ which is minimal over $I_{2}$ and does not contain $e$. But this is easily seen to be true using Chin's result (see 1.2): All prime ideals of $R$ minimal over $I_{2}$ are stable under the action of the connected component of $G$. Hence there are only a finite number of such minimal primes, and $G$ permutes them transitively. Therefore $M$ contains one of them, say $Q$, and $e \notin I_{2} \cap R^{G}=Q \cap R^{G}$.

As examples in $\S$ I.6 show, the assumptions on PI-degrees are necessary in the lemma. However, the requirement that $R^{G}$ be Artinian modulo a nilpotent ideal can be relaxed, because under certain hypotheses it is possible to reduce to this case by localization. This is the strategy for proving the next result.

Theorem 3.4. Assume either that $R$ is left Noetherian and char $k=0$, or else that $R$ is integral over its center. Let $P_{1}$ and $P_{2}$ be prime ideals of $R$ with the same PI-degree $d$ such that $\Phi\left(P_{1}\right) \cap \Phi\left(P_{2}\right) \neq \varnothing$. Then $\overline{P_{1} \cdot G} \cap \overline{P_{2} \cdot G} \neq \varnothing$. If every irreducible component of $\overline{P_{1} \cdot G} \cap \overline{P_{2} \cdot G}$ contains a prime ideal of PIdegree $d$, then $\Phi\left(P_{1}\right)=\Phi\left(P_{2}\right)$.

Example I.5.6 shows that this result can fail if $R$ is neither Noetherian nor integral over its center.

Proof. By I.6.12, $\overline{P_{1} \cdot G} \cap \overline{P_{2} \cdot G} \neq \varnothing$. Set $I_{\nu}=\bigcap_{g \in G} P_{\nu}^{g}$ for $\nu=1,2$. We may clearly assume that $I_{1} \cap I_{2}=0$. By 1.2 , each $I_{\nu}$ is a finite intersection of prime ideals. Since $\Phi\left(P_{1}\right) \cap \Phi\left(P_{2}\right) \neq \varnothing$, it follows by Lemma 1.1 that $0=P_{\nu} \cap C^{G}=$ $I_{\nu} \cap C^{G}$. Since $G$ permutes the minimal primes of $R$ over $I_{\nu}$ transitively, this implies that these minimal primes have zero intersection with $C^{G}$. So if $R$ is integral over its center, the localization result 2.5 applies, and if $R$ is Noetherian and char $k=0$, equidimensionality together with [MR, 6.3.11] implies that $\Phi\left(P_{1}\right) \cup \Phi\left(P_{2}\right)$ is the set of minimal prime ideals of $R^{G}$. Hence in this case we can localize by Corollary 2.4. One checks as in the proof of I.6.14 that localization preserves the data at hand, and that $\overline{S^{-1} P_{1} \cdot G} \cap \overline{S^{-1} P_{2} \cdot G}$ contains some prime ideal of PI-degree $d$. Therefore we may assume that $A^{G}$ is Artinian modulo its nilpotent nilradical. The theorem now follows from Lemma 3.3. (The assumption on the characteristic of $k$ is needed since the set $S$ in 2.4 is only known to be an Ore set in characteristic zero.)

We now give an alternative description of $\Phi$ for Azumaya algebras.

Theorem 3.5. Suppose $R=A$ is an Azumaya algebra with center $C$. Then for every prime ideal $P$ of $A, \Phi(P)=\left\{p \in \operatorname{Spec} A^{G} \mid p \cap C^{G}=P \cap C^{G}\right\}$.

We remark that the Azumaya algebra $A$ need not be Noetherian. Note that the inclusion " $\subseteq$ " holds by Lemma 1.1 for arbitrary PI-algebras.

Proof. The Azumaya algebra $A$ is a direct sum of Azumaya algebras $A_{d}$ of constant rank $d$. Moreover, each $A_{d}$ is $G$-stable. It follows easily that we may assume that $A$ is an Azumaya algebra of constant rank $d$.

Let $P_{1}$ be a prime ideal of $A$, and let $p$ be a prime of $A^{G}$ such that $P_{1} \cap C^{G}=p \cap C^{G}$. We have to show that $p \in \Phi\left(P_{1}\right)$. By Theorem 3.2, there exists some prime ideal $P_{2}$ of $A$ lying over $p$. If $\Phi\left(P_{1}\right) \cap \Phi\left(P_{2}\right) \neq \varnothing$, 
then Theorem 3.4 implies that $\Phi\left(P_{1}\right)=\Phi\left(P_{2}\right)$, so that $p \in \Phi\left(P_{1}\right)$, as desired. Hence we may assume that $\Phi\left(P_{1}\right) \cap \Phi\left(P_{2}\right)=\varnothing$. Note, however, that $P_{2} \cap C^{G}=$ $p \cap C^{G}=P_{1} \cap C^{G}$. We will derive a contradiction from this.

Let $I_{\nu}=\bigcap_{g \in G}\left(P_{\nu}\right)^{g}$. By Chin's result (see 1.2), $I_{\nu}$ is a finite intersection of prime ideals. Now $\bar{A}=A / I_{1} \cap I_{2}$ is an Azumaya algebra of constant rank $d$ with center $\bar{C}=C / C \cap I_{1} \cap I_{2}$. Note that $\Phi_{\bar{A}}\left(\bar{P}_{1}\right) \cap \Phi_{\bar{A}}\left(\bar{P}_{2}\right)=\varnothing$, and that $\overline{P_{\nu} \cap C^{G}}=\bar{P}_{\nu} \cap \bar{C}^{G}$. Replacing $A$ by $A / I_{1} \cap I_{2}$, we may therefore assume that $I_{1} \cap I_{2}=0$. Since $I_{1} \cap C^{G}=P_{1} \cap C^{G}=P_{2} \cap C^{G}=I_{2} \cap C^{G}$, it follows that $P_{\nu} \cap C^{G}=0$. By 1.2 , the localization result 2.5 applies. One checks easily that our assumptions on $P_{1}$ and $P_{2}$ are preserved under localization. Hence we may assume that $A^{G}$ is Artinian modulo its nilpotent nilradical. Now the condition $\Phi\left(P_{1}\right) \cap \Phi\left(P_{2}\right)=\varnothing$ means that $A^{G}=\left(P_{1} \cap A^{G}\right)+\left(P_{2} \cap A^{G}\right)=\left(I_{1} \cap A^{G}\right)+\left(I_{2} \cap A^{G}\right)$. It follows that $A=I_{1}+I_{2}$. Since $I_{1} \cap I_{2}=0, A=I_{1} \oplus I_{2}$. Note that $C=\left(C \cap I_{1}\right) \oplus\left(C \cap I_{2}\right)$, and that $F_{\nu} \supseteq I_{\nu}$. Since $P_{1} \cap C^{G}=P_{2} \cap C^{G}$, this implies that $P_{\nu} \cap C^{G} \supseteq C^{G}$, a contradiction to $P_{\nu} \subsetneq A$.

Let us consider the correspondence $\Phi: \operatorname{Spec} A \multimap \operatorname{Spec} A^{G}$. By the last result, the sets $\Phi(P)$ are either equal or disjoint. Hence $\Phi$ partitions Spec $A^{G}$. Therefore we can define an equivalence relation $\sim$ on $\operatorname{Spec} A^{G}$ by $p \sim q$ iff there is some $P$ such that both $p$ and $q$ belong to $\Phi(P)$. Denote the set of equivalence classes by $\operatorname{Spec} A^{G} / \Phi$, and let $\Phi^{\prime}$ be the map $\Phi^{\prime}: \operatorname{Spec} A \rightarrow \operatorname{Spec} A^{G} / \Phi$. Note that $\Phi^{\prime}$ is surjective by Theorem 3.2. We equip $\operatorname{Spec} A^{G} / \Phi$ with the quotient Zariski topology obtained from $\operatorname{Spec} A^{G}$. That is, a subset $X$ of $\operatorname{Spec} A^{G} / \Phi$ is closed iff

$$
\left\{p \in \operatorname{Spec} A^{G} \mid \exists P \in \operatorname{Spec} A \text { with } p \in \Phi(P) \text { and } \Phi^{\prime}(P) \in X\right\}
$$

is closed in $\operatorname{Spec} A^{G}$.

Consider the following diagram:

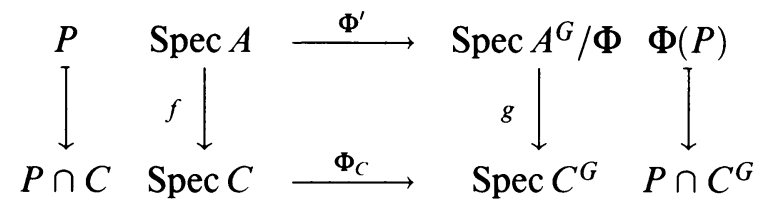

Here $\Phi_{C}$ is the natural map $\operatorname{Spec} C \rightarrow \operatorname{Spec} C^{G}$. It is well known that $f$ is a bijection and in fact a homeomorphism. By 3.5, $g$ is a bijection, and by I.6.19 it is a homeomorphism. Moreover, the diagram clearly commutes. We have proved:

Theorem 3.6. Let $R=A$ be an Azumaya algebra with center $C$. Then the correspondence $\Phi$ partitions $\operatorname{Spec} A^{G}$, so that one can form the set of equivalence classes $\operatorname{Spec} A^{G} / \Phi$. Moreover, the map $g: \operatorname{Spec} A^{G} / \Phi \rightarrow \operatorname{Spec} C^{G}$ sending $\Phi(P)$ to $P \cap C^{G}$ is a bijection and in fact a homeomorphism. Consider the above diagram. It commutes, and $f$ is a homeomorphism. In particular, since $\Phi_{C}$ is a continuous surjection sending closed G-invariant sets to closed sets, the same is true for $\Phi^{\prime}$.

Note that here $A$ is an arbitrary Azumaya algebra over $k$. 


\section{ACTIONS OF CONNECTED GROUPS BY SPEC-INNER AUTOMORPHISMS}

Throughout this section, $R$ is an arbitrary PI-algebra over $k$, and $H$ is a connected linear algebraic group acting rationally on $R$.

As in $\S I .9$, we say that an automorphism $g$ of $R$ is spec-inner iff all prime ideals are $g$-stable. It is clear that a spec-inner automorphism stabilizes all semiprime ideals. Moreover, if $I$ is a $g$-stable ideal of $R$, then $g$ induces a spec-inner automorphism of $R / I$. If $R$ is additionally affine and semiprime, then $g$ fixes the center of $R$ pointwise (I.9.1.1). This was heavily exploited in $\S I .9$. However, it is not true for arbitrary semiprime PI-algebras (for example, the field extension $k \subset k(x)$ has a nontrivial Galois group). But for a rational action of a connected linear algebraic group it is true, as we will see below. This will allow us in the next section to generalize many results from $\S$ I.9 to PI-algebras which are not necessarily affine.

Recall that an automorphism $g$ of a semiprime Goldie ring $A$ is called $X$-inner iff there is a nonzero element $x$ in the quotient ring of $A$ such that $x a^{g}=a x$ for all $a \in A$ (cf. [ $\mathrm{M}_{1}$, Chapter 3]). Note that inner automorphisms are $\mathrm{X}$-inner, and the $\mathrm{X}$-inner automorphisms of a prime PI-algebra are just those which fix the center pointwise. As mentioned above, a spec-inner automorphism of a prime PI-algebra need not fix the center pointwise; so spec-inner automorphisms need not be $\mathrm{X}$-inner, and even in the affine case, $\mathrm{X}$-inner automorphisms need not be spec-inner. But the situation is different for a rational action of a connected linear algebraic group-in this case the notions " $\mathrm{X}$-inner" and "spec-inner" are closely related and often equal.

Proposition 4.1. Let $R$ be a semiprime PI-algebra, and let $H$ be a connected linear algebraic group acting rationally on $R$ by spec-inner automorphisms. Then the center of $R$ is pointwise fixed under the action of $H$. In particular, if $R$ is prime, then $H$ acts by X-inner automorphisms.

Proof. Here we need two facts about rational actions of a connected group: First, any such action on a field is trivial, and second, any such action on a prime PI-algebra with nonzero Jacobson radical fixes the center pointwise (see the appendix). Let $x$ be an element in the center of $R$. It suffices to show that $x$ is fixed in every prime image of $R$. So we may assume that $R$ is prime. If the Jacobson radical of $R$ is nonzero, we are done. If not, it suffices to show that $x$ is fixed in all simple homomorphic images of $R$. So we may assume that $R$ is simple. But then the center of $R$ is a field and thus pointwise fixed.

Proposition 4.2. Let $H$ be a connected linear algebraic group acting rationally on a PI-algebra $R$. Assume that either $H$ is reductive, or that $R$ is Noetherian, or that $R$ is integral over its center.

(a) If $R$ is prime, then $H$ acts by spec-inner automorphisms iff it acts by $X$-inner automorphisms.

(b) More generally, $H$ acts by spec-inner automorphisms iff it acts by $X$ inner automorphisms on $R / P$ for each minimal prime ideal $P$ of $R$.

(c) If $R$ is semiprime with a finite number of minimal prime ideals, then $H$ acts by spec-inner automorphisms iff it fixes the center of $R$ pointwise.

Proof. In each part, the implication " $\Rightarrow$ " follows by Proposition 4.1.

(a) Suppose that $H$ acts by $\mathrm{X}$-inner automorphisms, and let $P$ be a prime 
ideal of $R$. Assume first that $R$ is integral over its center $C$. If $P$ is not $H$-stable, set $Q=\bigcap_{h \in H} P^{h}$. Then $Q$ is a semiprime ideal strictly contained in $P$. Moreover, since $H$ is connected, $Q$ is actually prime by a result of Chin [C] (see 1.2). (In the case when $R$ is Noetherian, this can be seen by elementary arguments, cf. I.2.19.) But $C \subseteq R^{H}$ so that $Q \cap C=P \cap C$, in contradiction to incomparability for the integral centralizing extension $C \subseteq R$. Hence $H$ acts by spec-inner automorphisms. Next suppose that $R$ is Noetherian. Then the action of $H$ on the trace ring $\mathrm{T}(R)$ is spec-inner since $\mathrm{T}(R)$ is integral over $\mathrm{Z}(\mathrm{T}(R))$. Using lying over for the finite centralizing extension $R \subseteq \mathrm{T}(R)$, we conclude that $H$ acts also on $R$ by spec-inner automorphisms.

Finally, assume that $H$ is reductive. Then it suffices to show that $P$ is stable under the action of every torus $T \subseteq H$. By I.9.4.2, such a $T$ contains a finite subgroup $F$ whose order is invertible in $k$ such that $R^{T}=R^{F}$. Given a prime $p$ in $R^{F}$ minimal over $P \cap R^{F}$, the only primes of $R$ lying over $p$ are the $F$-conjugates of $P\left(\right.$ see $\left[\mathrm{M}_{2}\right]$ or $\left.\left[\mathrm{M}_{3}\right]\right)$. Since all $T$-conjugates of $P$ lie over $p$, it follows that the $T$-orbit of $P$ is finite. Since $T$ is connected, $P$ is actually $T$-stable.

(b) By the above-mentioned result of Chin [C], all minimal primes of $R$ are $H$-stable. Hence (b) follows from (a).

(c) Suppose that $H$ fixes the center of $R$ pointwise. Denote by $\mathrm{Q}(R)$ the total ring of quotients of $R$, a central localization of $R$. Let $P_{1}, \ldots, P_{n}$ be the minimal prime ideals of $R$. Then the center of $\mathrm{Q}(R)$ is the direct sum of the centers of the $\mathrm{Q}\left(R / P_{i}\right)$. So for each $i$, the action of $H$ fixes the center of $\mathrm{Q}\left(R / P_{i}\right)$ pointwise and thus also the center of $R / P_{i}$. Hence $H$ acts on $R / P_{i}$ by $\mathrm{X}$-inner automorphisms. So by part (b), $H$ acts on $R$ by spec-inner automorphisms.

Let us recall two results from [I] which further clarify the situation. Their proofs go through nearly without change. First, assume that $R$ is a semiprime ring with a finite number of minimal prime ideals. Then $H$ acts on $R$ by $\mathrm{X}$-inner automorphisms iff there is some minimal prime $P$ of $R$ such that $H$ acts on $R / P$ by $\mathrm{X}$-inner automorphisms (I.9.1.6(a)). One should compare this with $4.2(\mathrm{~b})$.

Next assume that $R=A$ is a prime Azumaya algebra, and if char $k=$ $p \neq 0$, assume additionally that $H$ is reductive. Then if $H$ acts on $A$ by $\mathrm{X}$ inner automorphisms, it acts by inner automorphisms (I.9.1.6(c)). Generalizing example I.9.1.8, we now show that one cannot just drop the assumption here that $R=A$ is an Azumaya algebra. So actions by spec-inner (or X-inner) automorphisms are in fact more general than actions by inner automorphisms.

Example 4.3. Let $H$ be any linear algebraic group. Then there is a rational action of $H$ on an affine, prime, Noetherian PI-algebra $R$ finite over its center such that every nontrivial element of $H$ acts by a spec-inner automorphism which is not inner.

Being a linear algebraic group, $H$ embeds into $\mathrm{PGL}_{n}(k)$ for some $n$. Let $k[x]$ be a polynomial ring, and set

$$
R=k[x] \cdot I_{n}+\mathbf{M}_{n}(x k[x]) .
$$

Then $H$ acts rationally and faithfully on $R$, and the center of $R$ is pointwise fixed under the action of $H$. Proposition 4.2(a) implies therefore that $H$ acts 
by spec-inner automorphisms. Now let $h \in H$ act by conjugation by $a \in$ $\mathrm{GL}_{n}(k)$. Suppose there is an invertible element $b \in R$ such that $r^{h}=a^{-1} r a=$ $b^{-1} r b$ for all $r \in R$. Then $b a^{-1}$ is a central, invertible element in $\mathbf{M}_{n}(k[x])$ and therefore a scalar in $k$. Hence $b \in k \cdot a \subseteq \mathbf{M}_{n}(k)$. But $\mathbf{M}_{n}(k) \cap R=k \cdot I_{n}$, so $b$ is a scalar and $h$ is the identity element.

Remark 4.4. The last example shows also that a connected linear algebraic group acting by spec-inner automorphisms need not stabilize all ideals, even if $R$ is prime. Namely, suppose there are elements $h \in H$ and $a \in \mathrm{GL}_{n}(k)$ such that $a^{h} \notin k \cdot a$. (Actually, we can always arrange this by choosing a suitable embedding of $H$ into some $\mathrm{PGL}_{n}(k)$.) Set $J=k \cdot a x+\mathrm{M}_{n}\left(x^{2} k[x]\right)$. Then $J$ is an ideal of $R$ which is not $H$-stable. Of course, $J$ is not semiprime.

\section{ACTIONS OF LINEARLY REDUCTIVE GROUPS BY SPEC-INNER AUTOMORPHISMS}

Throughout this section, $R$ is an arbitrary PI-algebra over $k$, and $G$ is as usual a linearly reductive group acting rationally on $R$. We now study actions by spec-inner automorphisms.

Suppose that the characteristic of $k$ is prime, that $G$ is connected, and that $G$ acts by spec-inner automorphisms on a prime PI-algebra $R$. Then $G$ is a torus, and by I.9.4.2, there is a finite subgroup $F$ of $G$ whose order is invertible in $k$ such that $R^{G}=R^{F}$. Hence in prime characteristic, the results of this section can easily be deduced from the theory of actions of finite groups on noncommutative rings. However, this does not seem to be true in characteristic zero (cf. I.9.4).

If a finite group whose order is invertible acts on a semiprime ring, then the fixed ring is semiprime by a corollary to the theorem of Bergman and Isaacs [BI]. This is not true for actions by linearly reductive groups (see I.9.2.3). However, it is true in the setting at hand:

Lemma 5.1. Suppose that the connected component $G^{\circ}$ of $G$ acts by spec-inner automorphisms. Then given a semiprime ideal $I$ of $R, I \cap R^{G}$ is a semiprime ideal of $R^{G}$.

Proof. It clearly suffices to prove this in the case $I=P$ is prime. Then the center of $R / P$ is pointwise fixed under the action of $G^{\circ}$ by 4.1. Now I.9.2.1 applies and shows that $(R / P)^{G^{\circ}}=R^{G^{\circ}} / P \cap R^{G^{\circ}}$ is a semiprime ring. It follows by the theorem of Bergman and Isaacs that $P \cap R^{G}=\left(P \cap R^{G^{\circ}}\right) \cap R^{G}$ is a semiprime ideal.

Remark 5.2. One can actually prove a result which more closely resembles the theorem of Bergman and Isaacs. Namely, assume $R$ is semiprime with a finite number of minimal prime ideals, and that the action of $G^{\circ}$ fixes the center of $R$ pointwise (this means by 4.2 (c) that $G^{\circ}$ acts by spec-inner automorphisms). Then $I \cap R^{G} \neq 0$ for every $G$-stable right ideal $I \neq 0$ of $R$. However, my current proof of this seems unnecessarily complicated and is therefore omitted.

Next we consider chain conditions.

Lemma 5.3. Suppose that $R$ is semiprime, and that either the center of $R$ is pointwise fixed under the action of $G$, or that $G^{\circ}$ acts by spec-inner automor- 
phisms. Then if $R^{G}$ is left Noetherian, it is in fact two-sided Noetherian, and $R$ is a Noetherian left and right $R^{G}$-module.

Proof. If $G^{\circ}$ acts by spec-inner automorphisms, one easily reduces to the case that $G$ is connected, using 5.1 and a result by Farkas and Snider [FS]. So we may assume that the action of $G$ fixes the center of $R$ pointwise. The lemma follows now directly from [R, 5.1.6]. By the proof of the latter result, $R$ has only a finite number of minimal prime ideals. Hence $R^{G}$ is semiprime by I.9.2.1, and thus two-sided Noetherian by Cauchon's theorem. Now [R, 5.1.6] applies.

As in I.9.2, one can deduce some corollaries: If $R$ is semiprime and if either the center of $R$ is pointwise fixed or if $G^{\circ}$ acts by spec-inner automorphisms, then $R$ is Noetherian or Artinian iff $R^{G}$ is. Similarly, one can show that if $R$ is left Noetherian and if $G^{\circ}$ acts by spec-inner automorphisms, then $R$ is a Noetherian left $R^{G}$-module. The proof of the latter result proceeds as in I.9.2.7.

Another remark. If $G^{\circ}$ acts by spec-inner automorphisms, then $R$ is Schelter integral over $R^{G}$. The proof proceeds as in [MS]. (Note that after reducing to the case that $R$ is semiprime, the connected component of $G$ fixes the center $C$ of $R$ pointwise. Hence $C$ is then integral over $C^{G}$.) As a consequence, the results in [BV] apply to the situation at hand. In particular, the ring extension $R^{G} \subseteq R$ satisfies lying over. However, the latter result (and quite a bit more) can be proved directly:

Proposition 5.4 (Lying over). Suppose that $G$ acts on $R$ by spec-inner automorphisms. Then for every prime ideal $p$ of $R^{G}$ there is a unique prime ideal $P$ of $R$ lying over $p$. Moreover, $P$ contains every ideal $I$ of $R$ such that $I \cap R^{G} \subseteq p$.

As a corollary, one immediately obtains several versions of going up and going down (cf. I.9.3.7). Another consequence of this result is that for actions by spec-inner automorphisms, $\Phi$ partitions $\operatorname{Spec} R^{G}$. Hence we can, as in the case of Azumaya algebras, define a map $\Phi^{\prime}$ : Spec $R \rightarrow \operatorname{Spec} R^{G} / \Phi$. Under our current hypotheses, this map is bijective. In fact, it is a homeomorphism, if one endows $\operatorname{Spec} R^{G} / \Phi$ with the quotient Zariski topology (cf. I.9.3.6).

Proof. The proof proceeds as in I.9.3.2 and I.9.3.5. We show first that if $P_{1}$ and $P_{2}$ are distinct prime ideals of $R$, then $\Phi\left(P_{1}\right)$ and $\Phi\left(P_{2}\right)$ are disjoint; this will prove the uniqueness part of the proposition. Suppose to the contrary that there is a prime $p$ of $R^{G}$ which is minimal over both $P_{1} \cap R^{G}$ and $P_{2} \cap R^{G}$. Factoring out by $P_{1} \cap P_{2}$, we may assume that $P_{1} \cap P_{2}=0$. By Lemma 1.1, $P_{i} \cap C^{G}=p \cap C^{G}$, so that $P_{i} \cap C^{G} \subseteq P_{1} \cap P_{2}=0$. Hence the $G / G^{\circ}$-stable ideals $P_{i} \cap C$ of $C$ do not contain nonzero fixed points. The theorem of Bergman and Isaacs implies therefore that $P_{i} \cap C=0$. But every nonzero ideal of a semiprime PI-algebra intersects the center nontrivially. It follows that both $P_{1}$ and $P_{2}$ are 0 , whence equal, a contradiction.

Now let $p$ be a prime ideal of $R^{G}$, and let $I$ be an ideal of $R$ such that $I \cap R^{G} \subseteq p$. We want to find a prime ideal of $R$ lying over $p$ and containing $I$. Let $P$ be an ideal of $R$ maximal with respect to the properties that $I \subseteq P$ and $P \cap R^{G} \subseteq p$. Then $P$ is a prime ideal and as such $G$-stable. Factoring out by $P$, we may assume that $R$ is prime, and that for every nonzero ideal $J$ of $R, J \cap R^{G} \notin p$. We will show that $p$ is a minimal prime ideal of $R^{G}$. 
Suppose that $p \cap C^{G} \neq 0$, say $0 \neq x \in p \cap C^{G}$. Then $J=x R$ is a nonzero $G$-stable ideal of $R$, and $J \cap R^{G}=\natural(J)=x \natural(R)=x R^{G} \subseteq p$, a contradiction. Therefore $p \cap C^{G}=0$. Let $S$ be the set of nonzero elements of $C^{G}$. Since $C^{G}=C^{G / G^{\circ}}$, the domain $C S^{-1}$ is integral over the field $C^{G} S^{-1}$ and thus a field. Hence $R S^{-1}$ is the total ring of fractions of $R$ and thus Artinian. Therefore $\left(R S^{-1}\right)^{G}=R^{G} S^{-1}$ is Artinian. It follows that $p$ is a minimal prime ideal of $R^{G}$.

\section{APPENDIX: TRIVIALITY CRITERIA FOR RATIONAL ACTIONS}

The following triviality results should be well known, but are included for lack of a reference. I am grateful to R. Guralnick for some help with the part of the proofs dealing with the additive group $\mathbb{G}_{a}$.

Proposition A.1. Let $H$ be a connected linear algebraic group over $k$.

(a) Every rational action of $H$ on a field extension of $k$ is trivial.

(b) If char $k=0$ or if $H$ is reductive, also every rational action of $H$ on a division algebra over $k$ is trivial.

(c) Every rational action of $H$ on a commutative domain with nonzero Jacobson radical is trivial.

Before proving the proposition, let us deduce some corollaries.

Corollary A.2. If a connected linear algebraic group acts rationally on a prime PI-algebra with nonzero Jacobson radical, then it acts trivially on the center.

Proof. If $R$ is a prime PI-algebra with center $C$ and nonzero Jacobson radical, then $0 \neq \mathrm{Jac}(R) \cap C \subseteq \mathrm{Jac}(C)$.

Corollary A.3. If a connected linear algebraic group $H$ acts rationally on a semisimple Artinian algebra $R$ which is finite over its center, then it acts by inner automorphisms.

Proof. Since $H$ is connected, the simple components $R_{i}$ of $R$ are $H$-stable. By the proposition, $H$ acts trivially on the center of each $R_{i}$. Hence the Noether-Skolem theorem applies.

Proof of the proposition. We will show below that the proposition holds for the groups $\mathbb{G}_{m}$ and $\mathbb{G}_{a}$. Using this, we show now that it holds for every connected linear algebraic group $H$. Denote by $K$ the kernel of any of the above representations of $H$, i.e., $K$ is the closed subgroup consisting of those elements of $H$ which act trivially. Since every semisimple element of $H$ is contained in a torus, it follows that $H / K$ does not contain any semisimple elements; that is, $H / K$ is unipotent. So if $H$ is reductive, $H=K$, and if $H$ is not reductive, we use the fact that every nontrivial connected unipotent group contains a copy of $\mathbb{G}_{a}$. It follows again that $H=K$, so that in any case $H$ acts trivially.

Now let $H=\mathbb{G}_{m}$ act rationally on a commutative domain $R$ with nonzero Jacobson radical $J$. (Actually, $R$ need not be commutative here.) If the action on $R$ is nontrivial, then it is also nontrivial on $J$. Hence there is an element $x \in J$ which is a nontrivial eigenvector for $H$ : Every $\alpha \in H$ acts 
by $x^{\alpha}=\alpha^{n} \cdot x$ for some fixed integer $n \neq 0$. Since $k$ is algebraically closed, $x$ is transcendental over $k$. Since $x$ belongs to the Jacobson radical, the elements $x-\alpha^{-n}$ are invertible in $R$. Moreover, the elements $(1 /(x-1))^{\alpha}=$ $\alpha^{-n} /\left(x-\alpha^{-n}\right)$ of $R$ are all $k$-linearly independent, so that the action of $H$ is not locally finite, a contradiction. Hence $H=\mathbb{G}_{m}$ acts trivially on $R$. A similar argument shows also that $\mathbb{G}_{m}$ acts trivially on fields and division algebras.

Now let $H=\mathbb{G}_{a}$ act rationally on a commutative domain $R$ with nonzero Jacobson radical $J$. Suppose that the action of $H$ is nontrivial. Let $V$ be a nontrivial $H$-submodule of $J$ of minimal dimension over $k$, and denote by $\rho$ the representation of $H$ on $V$. Since $H$ is unipotent, $\rho$ can be brought into upper triangular form with 1's on the diagonal. The minimality assumption implies now that $\rho$ is actually of the form

$$
\rho=\left(\begin{array}{cc}
1 & \beta \\
0 & I_{r}
\end{array}\right)
$$

where $r=\operatorname{dim} V-1, \beta=\left(\beta_{1}, \ldots, \beta_{r}\right)$, and $I_{r}$ is the $(r \times r)$-identity matrix. Choosing suitable basis elements $x \in V$ and $y_{1}, \ldots, y_{r} \in V^{H}$, we have that for each $a \in H, \quad x^{a}=x+\beta_{1}(a) y_{1}+\cdots+\beta_{r}(a) y_{r}$. Let $k^{\prime}$ be the field extension of $k$ inside the field of fractions of $R$ generated by the $y_{i}$. Then $H$ acts trivially on $k^{\prime}$, and rationally on the algebra $R^{\prime}$ generated by $R$ and $k^{\prime}$. Now for each $a \in H, x^{a}=x+a(x)$ for some $a(x) \in k^{\prime}$. Since $\operatorname{ker} \beta=\operatorname{ker} \rho$ is finite, there are infinitely many such $a(x)$. This shows in particular that $x$ is transcendental over $k^{\prime}$, since $H$ embeds into the group of automorphisms of $k^{\prime}(x)$ over $k^{\prime}$. It follows easily that the $H$-orbit of $1 /(x+1)$ consists of infinitely many $k^{\prime}$-linearly independent elements, a contradiction to the fact that $H=\mathbb{G}_{a}$ acts rationally on $R^{\prime}$. Hence $\mathbb{G}_{a}$ acts trivially on $R$. Note that a similar argument shows also that $\mathbb{G}_{a}$ acts trivially on fields.

Finally, we assume that the characteristic of $k$ is zero. Let $R$ be a division algebra over $k$ with a rational action of $H=\mathbb{G}_{a}$. In the preceding paragraph, we used only at one point that $R$ was commutative, namely to obtain the field $k^{\prime}$ (one needs the fact that $x$ and the $y_{i}$ commute in order to prove that the $(1 /(x+1))^{a}$ are linearly independent). In characteristic zero, we can avoid this. We will see below that here $\rho$ is actually two-dimensional. Hence now $x^{a}=x+\beta(a) y$ and $(x / y)^{a}=(x / y)+\beta(a)$. The action on the field $k(x / y)$ is therefore as usual not rational, in contradiction to our original assumption.

So why is $V$ two-dimensional in characteristic zero? Let $a$ be any nonzero element of $H=\mathbb{G}_{a}$. Since the order of $a$ in $H$ is infinite, the subgroup generated by $a$ is dense in $H$. Therefore $\rho(a)$ acts nontrivially on $V$, and the Jordan normal form of $\rho(a)$ contains a nontrivial block with 1's on the diagonal. Hence there is a two-dimensional nontrivial $\rho(a)$-submodule $U$ of $V$. By a density argument, $U$ is $H$-stable. The minimality of $V$ now implies that $U=V$.

\section{REFERENCES}

[BI] G. M. Bergman and I. M. Isaacs, Rings with fixed-point-free group actions, Proc. London Math. Soc. 27 (1973), 69-87.

[BS] G. M. Bergman and L. M. Small, PI-degrees and prime ideals, J. Algebra 33 (1975), 435-462. 
[B] W. Borho, On the Joseph-Small additivity principle for Goldie rank, Comput. Math. 47 (1982), 3-29.

[BV] A. Braun and N. Vonessen, Integrality for PI-rings, J. Algebra (to appear).

[C] W. Chin, Actions of solvable algebraic groups on noncommutative rings, J. Contemp. Math. 124 (1992), 29-38.

[FS] D. Farkas and R. Snider, Noetherian fixed rings, Pacific J. Math 69 (1977), 347-353.

[JS] A. Joseph and L. W. Small, An additivity principle for Goldie rank, Israel J. Math. 31 (1978), 105-114.

[L] E. Letzter, Prime ideals in finite extensions of Noetherian rings, J. Algebra 135 (1990), 412-439.

[MR] J. C. McConnell and J. C. Robson, Noncommutative Noetherian rings, Wiley-Interscience, New York, 1988.

$\left[\mathrm{M}_{1}\right]$ S. Montgomery, Fixed rings of finite automorphism groups of associative rings, Lecture Notes in Math., vol. 818, Springer-Verlag, 1980.

[M $\left.\mathbf{M}_{2}\right]$, Prime ideals in fixed rings, Comm. Algebra 9 (1981), 423-449.

[M $\left.\mathrm{M}_{3}\right] \quad$ Prime ideals and group actions in non-commutative algebras, Contemp. Math. 88 (1989), 103-124.

[MS] S. Montgomery and L. W. Small, Integrality and prime ideals in fixed rings of P.I. rings, J. Pure Appl. Algebra 31 (1984), 185-190.

[R] L. H. Rowen, Polynomial identities in ring theory, Academic Press, New York, 1980.

[S] W. Schelter, Integral extensions of rings satisfying a polynomial identity, J. Algebra 40 (1976), 245-257; Errata, 44 (1977), 576.

[I, $\left.\mathrm{V}_{1}\right]$ N. Vonessen, Actions of linearly reductive groups on affine PI-algebras, Mem. Amer. Math. Soc. No. 414 (1989).

$\left[\mathrm{V}_{2}\right] \quad$, Prime ideals in intermediate integral centralizing extensions of PI-rings, Comm. Algebra 19 (1991), 795-802.

[W] R. B. Warfield, Prime ideals in ring extensions, J. London Math. Soc. 28 (1983), 453-460.

Department of Mathematics, DRB 155, University of Southern California, Los AnGELES, CALIFORNIA 90089-1113

E-mail address: vonessen@math.usc.edu 